\title{
Fermentation of glycerol to 1,3-propanediol by Klebsiella and Citrobacter strains
}

\author{
Thomas Homann", Carmen Tag1, Hanno Biebl ${ }^{1}$, Wolf-Dieter Deckwer' ${ }^{1}$, and Bernhard Schink ${ }^{2}$ \\ 1 GBF-Gesellschaft für Biotechnologische Forschung mbH, Mascheroder Weg 1, D-3300 Braunschweig, Federal Republic of \\ Germany \\ ${ }^{2}$ Lehrstuhl für Mikrobiologie I, Universität Tübingen, Auf der Morgenstelle 28, D-7400 Tübingen, Federal Republic of Germany
}

Summary. Glycerol-fermenting anaerobes were enriched with glycerol at low and high concentrations in order to obtain strains that produce 1,3-propanediol. Six isolates were selected for more detailed characterization; four of them were identified as Citrobacter freundii, one as Klebsiella oxytoca and one as K. pneumoniae. The Citrobacter strains formed 1,3-propanediol and acetate and almost no by-products, while the Klebsiella strains produced varying amounts of ethanol in addition and accordingly less 1,3-propanediol. Enterobacterial strains of the genera Enterobacter, Klebsiella, and Citrobacter from culture collections showed similar product patterns except for one group which formed limited amounts of ethanol, but no propanediol. Seven strains were grown in $\mathrm{pH}$-controlled batch cultures to determine the parameters necessary to evaluate their capacity for 1,3-propanediol production. K. pneumoniae DSM 2026 exhibited the highest final concentration $(61 \mathrm{~g} / 1)$ and the best productivity $(1.7 \mathrm{~g} / 1 \mathrm{~h})$ whereas $C$. freundii $\mathrm{Zu}$ and $\mathrm{K} 2$ achieved only $35 \mathrm{~g} / 1$ and $1.4 \mathrm{~g} / 1 \mathrm{~h}$, respectively. The Citrobacter strains on the other hand gave somewhat better yields which were very close to the theoretical optimum of $65 \mathrm{~mol} \%$.

\section{Introduction}

As a glycol, 1,3-propanediol can principally be utilized as a monomer for the synthesis of polyesters and polyurethanes. The polymers on the basis of 1,3-propanediol are considered to exhibit better product properties and greater light stability than those produced from 1,2-propanediol, butanediols, or ethylene glycol (Elm et al. 1980). 1,3-Propanediol is synthesized at present in only small amounts from acrolein. The relatively high price, which is due to costly distillation, has prevented its application in polymer industries up to now. If $1,3-$ propanediol could be produced more cheaply by a fer- mentation process its use for the synthesis of plastics with specific properties might be envisaged. Glycerol as the chemical feedstock could be supplied as diluted raw glycerol, which is released from fat saponification for soap and detergent manufacture.

Fermentation of glycerol occurs rarely in rather different bacterial groups. It is oxidized via dihydroxyacetone to glyceraldehyde-3-phosphate and further via pyruvate to acetate, formate, $\mathrm{CO}_{2}$ and other products. The reducing equivalents may be transferred to an external electron acceptor or to the dehydration product of glycerol, 3-hydroxypropionaldehyde. The final product, 1,3-propanediol, is highly specific for glycerol fermentation and cannot be obtained from any other anaerobic conversion.

Among the Enterobacteriaceae, anaerobic growth with glycerol and formation of 1,3-propanediol is used as a character for identification of the genus Citrobact$e r$, but is also found in strains of Klebsiella (Toraya et al. 1980). The products of glycerol oxidation arise from pyruvate cleavage to acetyl coenzyme $A$ and formate, resulting in excretion of ethanol, acetate, $\mathrm{CO}_{2}$ and $\mathrm{H}_{2}$ (Mickelson and Werkman 1940). Streekstra et al. (1987) have recently investigated the energetic aspects of glycerol fermentation to 1,3-propanediol in chemostat cultures of $K$. pneumoniae.

Several Clostridium species also form 1,3-propanediol, together with butyric and acetic acid and some butanol or lactic acid (Nakas et al. 1983; Forsberg 1987). Some lactic acid bacteria form 1,3-propanediol from glycerol if a growth and energy substrate is present in the medium (Schütz and Radler 1984). Recently strictly anaerobic non-spore-forming, glycerol-utilizing rods have been described which ferment glycerol in a disproportionation reaction to 1,3-propanediol and 3-hydroxypropionic acid (Schink and Stieb 1983; Stieb and Schink 1984). Nothing is known about their fermentation capacity.

Suitable organisms for a glycerol fermentation process might be looked for among established strains of the genera Citrobacter, Klebsiella, or Clostridium. However, up to now no effort to investigate the natural 
potential for 1,3-propanediol-producing organisms has been published. It was the purpose of this study to obtain the bacteria representing this fermentation type from anaerobic enrichment cultures, to compare them with existing glycerol-fermenting strains, and to evaluate them for technical applications.

\section{Materials and methods}

Media and growth conditions. Enrichment cultures from sediments and sewage sludge with $0.09 \%$ glycerol were performed in a bicarbonate-buffered, sulphide-reduced mineral medium (Schink and Pfennig 1982). Enrichment cultures with $2 \%$ glycerol, precultures, and cultures for preliminary tests were grown in $100-\mathrm{ml}$ screwcapped bottles with rubber septa for syringe operation. They were filled with $30 \mathrm{ml}$ preboiled medium and sealed under nitrogen before autoclaving. The medium contained in $\mathrm{g} / 1$ deionized water: glycerol, $20 \mathrm{~g} ; \mathrm{K}_{2} \mathrm{HPO}_{4}, 3.4 \mathrm{~g} ; \mathrm{KH}_{2} \mathrm{PO}_{4}, 1.3 \mathrm{~g} ;\left(\mathrm{NH}_{4}\right)_{2} \mathrm{SO}_{4}, 2 \mathrm{~g}$; $\mathrm{MgSO}_{4} \cdot 7 \mathrm{H}_{2} \mathrm{O}, 0.2 \mathrm{~g} ; \mathrm{CaCl}_{2} \cdot 2 \mathrm{H}_{2} \mathrm{O}, 0.02 \mathrm{~g} ; \mathrm{FeSO}_{4} \cdot 7 \mathrm{H}_{2} \mathrm{O}, 5 \mathrm{mg}$; yeast extract, $1 \mathrm{~g}$; trace element solution SL 7 (Biebl and Pfennig 1981), $2 \mathrm{ml}$; $\mathrm{CaCO}_{3}, 2 \mathrm{~g}$. The incubation temperature was $30^{\circ} \mathrm{C}$.

For growth and product formation experiments a $1 \mathrm{~m}$ fermentor of BCC, Göttingen, FRG, was used. It was equipped with a control unit to adjust the $\mathrm{pH}$ to 7,0 and the agitation speed to 150 $\mathrm{rpm}$. The temperature was maintained at $32^{\circ} \mathrm{C}$ using an external thermostat. Slight gassing with purified nitrogen provided anaerobic conditions. The medium for fermentor cultures was amended to contain only $1 \mathrm{~g} \mathrm{~K}_{2} \mathrm{HPO}_{4}$ and $0.5 \mathrm{~g} \mathrm{KH}_{2} \mathrm{PO}_{4}$, and no $\mathrm{CaCO}_{3}$

Isolation. Pure cultures were obtained by repeated application of the agar shake culture method (Pfennig 1978) using screw-capped test tubes which were gassed with $\mathrm{N}_{2}$. Purified isolates were kept semi-aerobically on agar slants.

Analytical techniques. The fermentation products were separated and determined gas chromatographically on a Chromosorb 101 column of $1 \mathrm{~m}$ length installed in a Packard instrument, Model 408A (Chrompack, Frankfurt/M, FRG). The column temperature was programmed from $150^{\circ} \mathrm{C}$ to $220^{\circ} \mathrm{C}$ in several steps; the carrier gas was nitrogen. Isobutanol was used as internal standard.

Glycerol appeared after the fermentation products at the end of the chromatograms as a broad peak which allowed a rough estimation of its concentration. Quantitative determination was performed enzymatically via glycerokinase and L-lactate dehydrogenase using the test kit and instructions of Boehringer, Mannheim, FRG. D-Lactate was also measured by a Boehringer test operating with $D$-lactate dehydrogenase and glutamate-pyruvate transaminase.

Optical density was measured at $650 \mathrm{~nm}$ in a $1-\mathrm{cm}$ cuvette. Cell dry weights were obtained from washed centrifuged pellets dried at $80^{\circ} \mathrm{C}$ for $24 \mathrm{~h}$.

For identification of isolated Enterobacteriaceae strains the Enterotube II test kit of F. Hoffmann La Roche (Basel, Switzerland) was applied.

\section{Results}

\section{Enrichment and isolation}

Enrichment with $0.09 \%$ glycerol. Half-filled $120-\mathrm{ml}$ serum bottles were inoculated each with $5 \mathrm{ml}$ of anoxic black sediment or sewage sludge samples, and closed under a $\mathrm{N}_{2} / \mathrm{CO}_{2}$ mixture $(90 \% / 10 \%)$ with butyl rubber stoppers. Growth and gas production started after 1-5 days, and subsequent transfers grew up within 1-2 days. Purification of the prevalent bacteria by agar shake culture yielded the strains $\mathrm{Zu}$ from a sugar factory sewage sludge (Nörten-Hardenberg, FRG), Gö from the municipal sewage treatment plant in Göttingen, FRG, and Lin from a polluted creek near Hannover, FRG.

Enrichment with $2 \%$ glycerol. Four anaerobic culture bottles were inoculated with samples of garden compost, pond sediment and sludge (fresh and activated) of the Wolfenbüttel sewage plant (FRG). After 1 or 2 days, growth and 1,3-propanediol formation occurred in each of them. From the sewage culture, strain Bs2e was isolated and corresponded to strain Lin in cell shape and colony size. From the compost was obtained strain $\mathrm{K} 2$ and from the pond sediment strain $\mathrm{T} 3$, which both corresponded to strains $\mathrm{Zu}$ and Gö. All six strains produced 1,3-propanediol. A third group of isolates which grew weakly and formed only ethanol in low amounts was not further regarded.

Although conditions were anaerobic throughout we did not obtain clostridia from any of the enrichments.

\section{Identification}

All six isolates obtained were rod-shaped. Strains Lin and $\mathrm{Bs} 2 \mathrm{e}$ had rather large, but somewhat varying cells of $0.8-1.4 \times 2-6 \mu$ in size when grown on glycerol and were immotile. The other strains measured only 0.6 $0.9 \times 1.3-4 \mu$ and were strikingly motile. Negative contrast electron micrographs revealed peritrichous flagellation.

All strains grew aerobically and gave a negative oxidase reaction. The products of glucose fermentation were predominantly 2,3-butanediol and ethanol with the immotile strains and acetic and lactic acid with the motile ones. A biochemical test system allowed classification of the immotile strains as Klebsiella sp. and the motile ones as Citrobacter freundii. Strain Lin was identified as $K$. oxytoca, and strain $\mathrm{Bs} 2 \mathrm{e}$ as $K$. pneumoniae because of a positive indole reaction. The determination of strain Bs2e was substantiated by additional tests, i.e. a positive fecal coliform reaction, no growth at $10^{\circ} \mathrm{C}$ and no utilization of melizitose and gentisate.

\section{Fermentation products from glycerol}

Isolates. Table 1 shows the fermentation patterns of the isolates when grown in a $2 \%$ glycerol medium with $\mathrm{CaCO}_{3}$ buffering. While the Klebsiella strains produced predominantly ethanol ( $\mathrm{Lin})$ or both ethanol and acetate (Bs2e) in addition to 1,3-propanediol, the Citrobacter strains fermented glycerol almost entirely to $1,3-$ propanediol and acetate.

Except for strain Bs2e the fermentation ceased after consumption of $80-90 \mathrm{mmol} / 1$ glycerol at a $\mathrm{pH}$ of about 4.8. Glycerol was completely used up when the culture $\mathrm{pH}$ was regularly readjusted to 7.0 (not shown). When the growth temperature was raised to $37^{\circ} \mathrm{C}$, fer- 
Table 1. Anaerobic utilisation of glycerol by isolates in a $\mathrm{CaCO}_{3}$-buffered medium

\begin{tabular}{|c|c|c|c|c|c|c|}
\hline \multirow[t]{2}{*}{ Species } & \multirow[t]{2}{*}{ Strain } & \multirow{2}{*}{$\begin{array}{l}\text { Glycerol } \\
\text { consumed } \\
(\mathrm{mmol} / \mathrm{l})\end{array}$} & \multicolumn{4}{|c|}{ Products formed $(\mathrm{mmol} / 1)$} \\
\hline & & & $\begin{array}{l}\text { 1,3-Pro- } \\
\text { panediol }\end{array}$ & Ethanol & $\begin{array}{l}\text { Acetic } \\
\text { acid }\end{array}$ & $\begin{array}{l}\text { Lactic } \\
\text { acid }\end{array}$ \\
\hline Klebsiella oxytoca & Lin & 92 & 31 & 24 & 3 & ++ \\
\hline K. pneumoniae & Bs2e & 158 & 81 & 19 & 12 & + \\
\hline Citrobacter freundii & $\mathrm{Zu}$ & 86 & 41 & 1 & 12 & + \\
\hline C. freundii & Gö & 91 & 42 & 1 & 12 & + \\
\hline C. freundii & $\mathrm{T} 3$ & 76 & 33 & 1 & 11 & + \\
\hline C. freundii & $\mathrm{T} 2$ & 79 & 43 & 3 & 13 & + \\
\hline
\end{tabular}

Table 2. Anaerobic utilization of glycerol by selected Enterobacteriaceae strains from culture collections

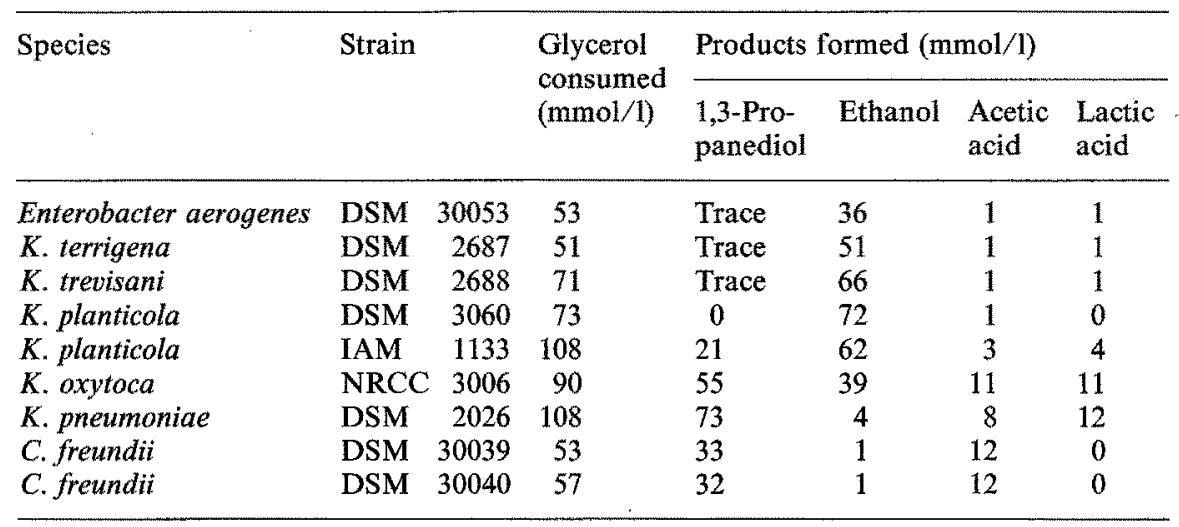

Glycerol was not fermented by E. cloacae DSM 30054 and DSM 30056 and by Bacillus polymyxa DSM 36 and NRCC 9053

mentation was markedly slower in $K$. oxytoca Lin and C. freundii $\mathrm{Zu} ; \mathrm{C}$. freundii Gö did not grow at $37^{\circ} \mathrm{C}$.

Collection strains. Enterobacterial strains as well as two bacilli from culture collections exhibited rather different fermentation patterns (Table 2). Two strains of Enterobacter cloacae and the two bacilli did not ferment glycerol at all. One Enterobacter and three Klebsiella strains of different species formed limited amounts of ethanol but no propanediol. Three other Klebsiella strains formed propanediol in addition to ethanol and acetate, the proportion of which varied considerably. The two $C$. freundii strains behaved as the isolated ones, 1,3-propanediol and acetate being almost the only products.

\section{PH-controlled glycerol fermentations}

Three Klebsiella and four Citrobacter strains were selected to be grown in $\mathrm{pH}$-controlled fermentor cultures with $2 \%$ glycerol. The final results listed in Table 3 do

Table 3. Fermentation of $2 \%$ glycerol in a $1-1$ fermentor at $\mathrm{pH} 7.0$

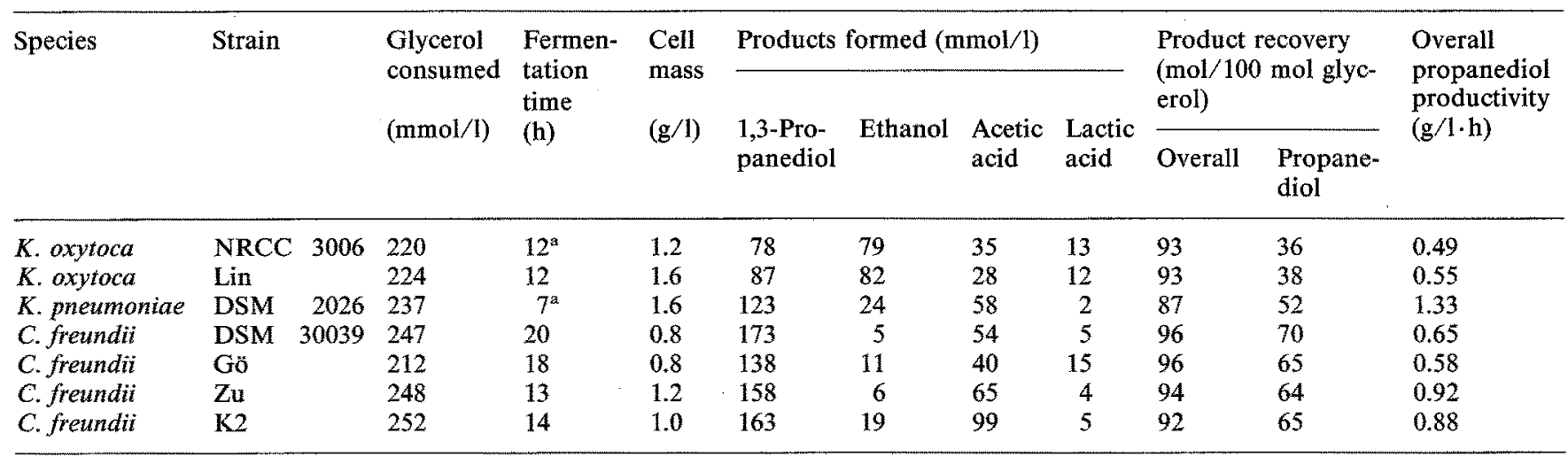

a Growth temperature $37^{\circ}$ instead of $32^{\circ} \mathrm{C}$ 


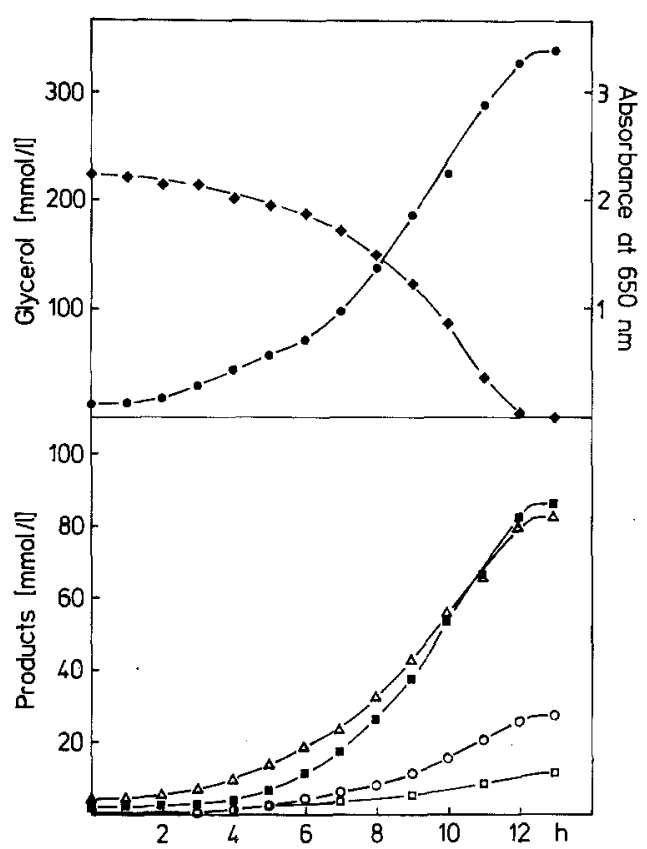

Fig. 1. Fermentation of $2 \%$ glycerol by Klebsiella oxytoca Lin at $32^{\circ} \mathrm{C}$ and $\mathrm{pH}$ 7.0: $\bullet$, absorbance; $\bullet$, glycerol; $\mathbf{\mathbf { 0 }}, 1,3$-propanediol; $\triangle$, ethanol; $O$, acetic acid; $\square$, lactic acid

not differ too much from those of the bottle cultures (Tables 1 and 2), but they permit a better recognition of the fermentation balance. The two $K$. oxytoca strains behaved very similarly: they formed a relatively high amount of ethanol and consequently less 1,3-propanediol and acetic acid. In the Citrobacter cultures there was some ethanol and lactic acid, too, at the end of the fermentation, but acetic acid and 1,3-propanediol were by far the predominating products. $K$. pneumoniae DSM 2026 exhibited a similar product composition, but the propanediol yield was markedly lower than with $\mathrm{Ci}$ trobacter.

The fermentation time for $2 \%$ glyerol was somewhat shorter with $K$. oxytoca than with Citrobacter, but due to the higher yields, the overall productivity for propanediol was superior with the Citrobacter strains. Highest productivity was achieved by $K$. pneumoniae DSM 2026 because of its unusually high fermentation rate.

In Figs. 1 and 2 the kinetics of growth, substrate uti-

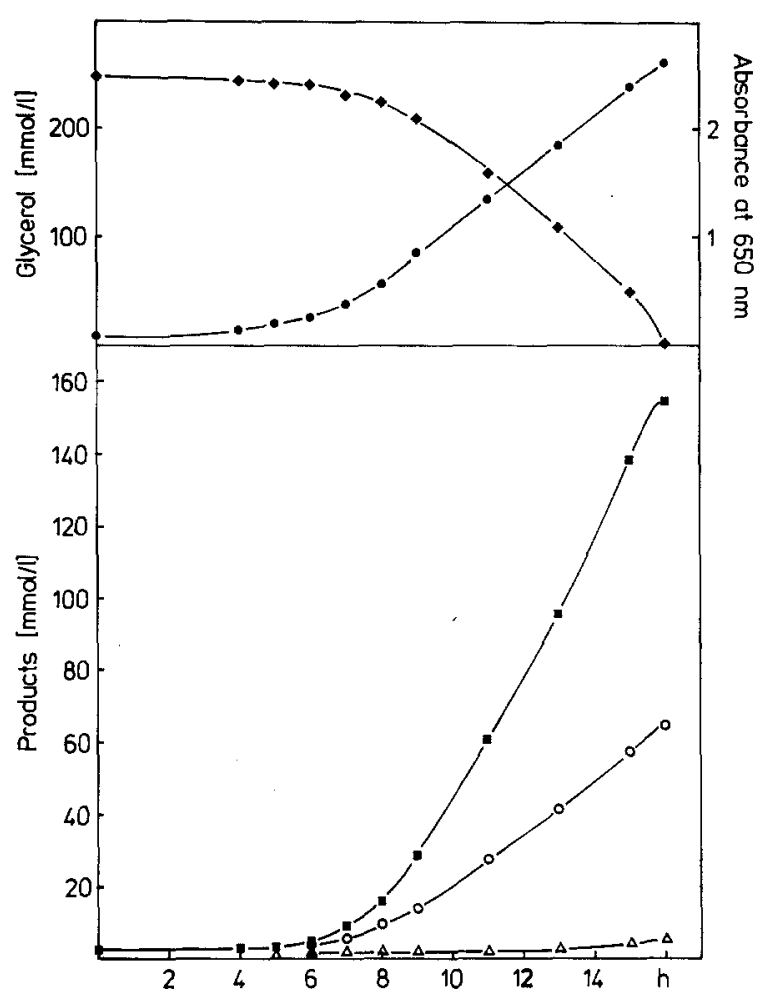

Fig. 2. Fermentation of $2 \%$ glycerol by Citrobacter freundii $\mathrm{Zu}$ at $32^{\circ} \mathrm{C}$ and $\mathrm{pH}$ 7.0: symbols as in Fig. 1

lization and product formation are shown for one strain of each group. Cultures of Citrobacter never grew without a delay of 3-6 h, during which time the cells elongated considerably. The exponential growth phase lasted about $6 \mathrm{~h}$ for all strains and was followed by a phase of linear growth. There was no shift of product formation with the growth phases; all products increased in a fixed proportion.

As shown in Table $45 \%$ glycerol was also completely fermented (the two slow-growing Citrobacter strains were not included). The product composition did not change with Citrobacter; with Klebsiella the proportions shifted somewhat from ethanol to propanediol and acetate. The calculated 1,3-propanediol productivity was higher with $5 \%$ than with $2 \%$ glycerol in all five cultures.

Table 4. Fermentation of $5 \%$ glycerol in a 1-1 fermentor at $\mathrm{pH} 7.0$

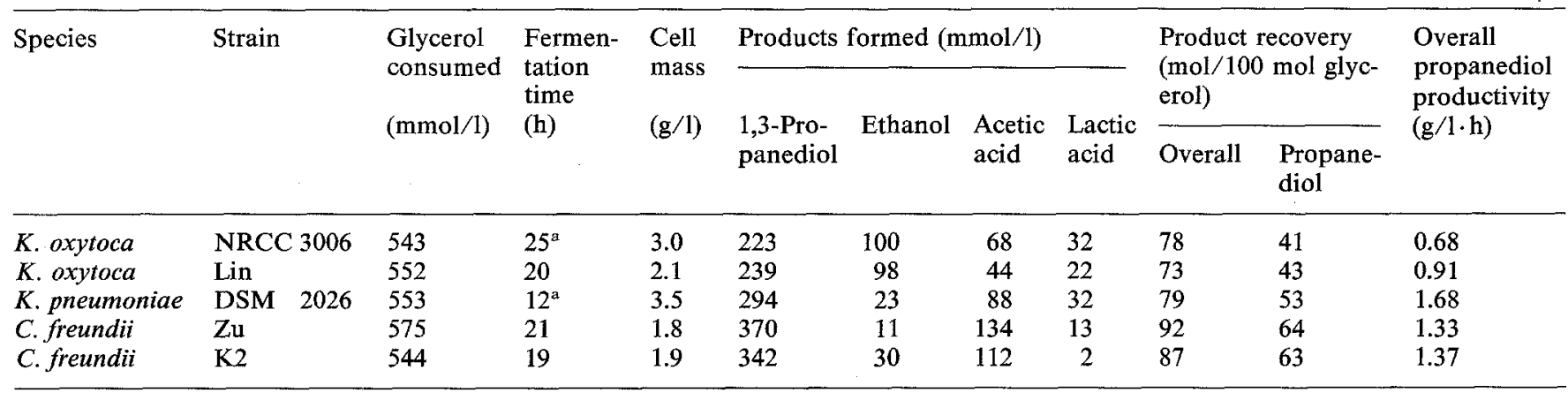

a Growth temperature $37^{\circ}$ instead of $32^{\circ} \mathrm{C}$ 
A glycerol concentration of $10 \%$ was used only by K. pneumoniae DSM 2026, which also fermented $12 \%$ glycerol. With the other strains, best glyerol utilization was obtained with $C$. freundii $\mathrm{K} 2$ with $63 \mathrm{~g} / 1$, while $K$. oxytoca Lin did not grow at all. Strain K2 fermented $7 \%, 9 \%$ and $10 \%$ glycerol with about the same result indicating that the maximum fermentation capacity is mainly determined by product inhibition.

\section{Discussion}

Among the strains isolated from enrichment cultures with glycerol and the collection strains screened for glycerol utilization, different fermentation types were recognized. One group including an Enterobacter and several Klebsiella strains as well as some unidentified isolates, is characterized by formation of ethanol as the only product and by limited glycerol turnover. Although the fermentation balance is theoretically compensated in the case of ethanol formation from glycerol, there are some reduction equivalents released during cell mass formation which are obviously transferred to external electron acceptors such as yeast extract components present in the medium (Thimann 1963). The other glycerol utilizers transfer the surplus reducing equivalents to further glycerol molecules releasing 1,3propanediol as a fermentation product. This type is represented by a number of Klebsiella species on the one hand and Citrobacter on the other.

The majority of the Klebsiella strains forms greater proportions of ethanol and accordingly less 1,3-propanediol while Citrobacter performs an almost pure 1,3propanediol/acetic acid fermentation of glycerol. A very low final ethanol and fairly high 1,3-propanediol content is also present in cultures of $K$. pneumoniae strain DSM 2026 (=NCTC 418) and strain Bs2e. However, the ethanol proportion increases with DSM 2026 under conditions of energy substrate limitation (Streekstra et al. 1987; unpublished results), whereas glycerol fermentation of Citrobacter seems to be almost uninfluenced by the culture conditions.

No organisms have been found which form ethanol as the main product in addition to a small amount of 1,3-propanediol just to compensate for the redox level difference between glycerol and cell material. This means that propanediol is always produced concomitantly with acetic acid. Streekstra et al. (1987) found the minimum of 1,3-propanediol excretion which appeared under glycerol limitation in cultures of $K$. pneumoniae DSM 2026 to remain at a relatively high level, and they inferred that the two enzymes involved in glycerol reduction have a higher affinity to the substrate than the oxidizing enzyme.

Clostridia were not enriched from the cultures described, but they invariably appeared when the samples were pasteurized prior to inoculation (Widdel, personal communication, unpublished observations). This might indicate a selective advantage for enteric bacteria in the habitats considered or an absence of vegetative Clostridium cells in the samples.
For evaluation of a fermentation process for technical application one has to consider the yield of the product desired, the substrate turnover rate and the final product concentration, i.e. the tolerance of the organism towards its products. Among the investigated strains, the highest yield of 1,3-propanediol was achieved by Citrobacter which approached the theoretical maximum:

$$
\begin{aligned}
\text { Glycerol } & \rightarrow 0.33 \text { acetate }+0.671,3 \text {-propanediol } \\
& +0.33 \mathrm{H}_{2}+0.33 \mathrm{CO}_{2} .
\end{aligned}
$$

If the cell mass production, which was estimated from the batch cultures to be around 5\% of the glycerol utilized, is taken into account the 1,3-propanediol yield does not change significantly due to the redox level difference between glycerol and cell mass:

$$
\begin{aligned}
\text { Glycerol } & \rightarrow 0.3 \text { acetate }+0.65 \text { 1,3-propanediol } \\
& +0.3 \mathrm{H}_{2}+0.3 \mathrm{CO}_{2}+0.05\left[\mathrm{C}_{4} \mathrm{H}_{8} \mathrm{O}_{3}\right] .
\end{aligned}
$$

However, depending on the strain and the culture conditions, the by-products in Citrobacter cannot be neglected and vary from $2 \%$ to $7 \%$ for ethanol and $1 \%$ to $8 \%$ for lactic acid.

The 1,3-propanediol yield of $K$. pneumoniae was lower than that of Citrobacter and amounted only to $0.53 \mathrm{~mol} / \mathrm{mol}$ glycerol for strain DSM 2026 in contrast to $0.65 \mathrm{~mol}$ for Citrobacter. However, due to its rapid fermentation the resulting productivity of $K$. pneumoniae for propanediol is considerably higher. As DSM 2026 in addition exhibits a product tolerance which is twice as high as with Citrobacter, this strain must be regarded as the most suitable one for the development of a 1,3-propanediol fermentation process at present. However, since $K$. pneumoniae is regarded as an opportunistic pathogen and thus may give rise to safety discussions the classical "trimethyleneglycol" formers of the genus Citrobacter should be kept in mind for future research activities in fermentative 1,3-propanediol production.

Acknowledgements. We thank Rolf Schauder for his contribution in strain isolation, Heinrich Lünsdorf for negative contrast electron micrographs and Sabine Marten for excellent technical assistence.

\section{References}

Biebl H, Pfennig N (1981) Isolation of members of the family Rhodospirillaceae. In: Starr MP, Stolp H, Trüper HG, Balows A, Schlegel HG (eds) The prokaryotes. Springer, Berlin, Heidelberg, New York

Elm R, Falbe J, Hahn H-D, Gelbke H-P (1980) Propandiole. In: Bartholomé E, Biekert E, Hellmann $H$, Ley $H$, Weigert $M$, Weise E (eds) Ullmanns Encyklopädie der technischen Chemie, vol. 19. Verlag Chemie, Weinheim

Forsberg CW (1987) Production of 1,3-propanediol from glycerol by Clostridium acetobutylicum and other Clostridium species. Appl Environ Microbiol 53:639-643

Mickelson MN, Werkman CH (1940) The dissimilation of glycerol by coli-aerogenes intermediates. J Bacteriol 39:709-715

Nakas JP, Schaedle M, Parkinson CM, Coonley CE, Tanenbaum SW (1983) System development of linked-fermentation production of solvents from algal biomass. Appl Environ Microbiol 46:1017-1023 
Pfennig N (1978) Rhodocyclus purpureus gen. nov. sp. nov., a ringshaped, vitamin $\mathrm{B}_{12}$-requiring member of the family Rhodospirillaceae. Int J Syst Bacteriol 28:283-288

Schink B, Pfennig N (1982) Fermentation of trihydroxybenzenes by Pelobacter acidigallici gen. nov. sp. nov., a new strictly anaerobic, non-spore-forming bacterium. Arch Microbiol 133:195201

Schink B, Stieb M (1983) Fermentative degradation of polyethylene glycol by a strictly anaerobic, Gram-negative, non-sporeforming bacterium, Pelobacter venetianus sp. nov. Appl Envirion Microbiol 45:1905-1913

Schütz H, Radler F (1984) Anaerobic reduction of glycerol to propanediol-1,3 by Lactobacillus brevis and Lactobacillus buchneri. Syst Appl Microbiol 5:169-178
Stieb M, Schink B (1984) A new 3-hydroxybutyrate fermenting anaerobe, Ilyobacter polytropus gen. nov. sp. nov., possessing various fermentation pathways. Arch Microbiol 140:139-146

Streekstra H, Teixeira de Mattos MJ, Neijssel OM, Tempest DW (1987) Overflow metabolism during anaerobic growth of Klebsiella aerogenes NCTC 418 on glycerol and dihydroxyacetone in chemostat culture. Arch Microbiol 147:268-275

Thimann KV (1963) The life of bacteria, 2nd edn. The MacMillan Company, New York

Toraya T, Kuno S, Fukui S (1980) Distribution of coenzyme $B_{12^{-}}$ dependent diol dehydratase and glycerol dehydratase in selected genera of Enterobacteriaceae and Propionibacteriaceae. J Bacteriol 141:1439-1442 